\title{
Human genetics around the world
}

This issue of Genetics in Medicine introduces a new section on International Genetics. As part of its mission to promote the application of genetics and genetic education in all areas of medicine, Genetics in Medicine takes the initiative to broaden the horizon, and increase the awareness, of College members. The realization that goals and obstacles are largely shared by geneticists around the world enhances our sense of community and of global responsibilities. The section on International Genetics will provide an avenue for international exchange and interaction.

This effort aligns perfectly with the goals of the International Federation of Human Genetics Societies (IFHGS), founded in 1996 as an umbrella organization of organized groups dedicated to all aspects of human genetics, including research, clinical practice, and professional and lay education. As stated in the IFHGS bylaws, "the Federation will enable communication between its member groups and encourage interaction between workers in genetics fields and in related sciences and will make itself available to promote meetings and publications and other forums which support human genetics research and practice."

The Federation has different levels of membership. The three founding multinational societies are Full Members: the American Society of Human Genetics (ASHG), the European Society of Human Genetics (ESHG), and the Human Genetics Society of Australasia (HGSA). New multinational or regional societies are currently being formed in South/Central America and South East Asia, that will then apply to the Federation for Full Member status. National Human or Medical Genetics Societies are eligible to participate in the Federation as Corresponding Members. Currently, there are 36 Corresponding Member Societies spanning the globe. All participating individuals, whether from Full or Corresponding Member Societies, are elected officers or long-term representative appointees of the constituent human genetics societies. Representatives of Full Member Societies comprise the Executive Committee.

The Federation maintains a website at http://www.ifhgs.org/ with updated information on Member Societies: contacts, mailing addresses, web addresses, policy statements, and endorsements. Member Societies post annual meeting information; currently, there are 19 regional meetings listed for 2001. The homepage also contains IFHGS membership information and application forms and a link to the 10th International Congress of Human Genetics (ICHG) to be held in May 2001 in Vienna, Austria, which will be the first IFHGS-sponsored International Congress. At the 9th ICHG in Brazil, representatives of the Federation decided to undertake the organization and support of future International Congresses that are held at 5-year intervals. Sponsorship and organizational responsibilities rotate among the full member Societies who will subsume their annual meeting into the Congress. This policy will guarantee high attendance, fiscal soundness, and geographic rotation of the International Congresses. While the 10th ICHG is organized by the ESHG, the 11th ICHG, to be held in 2006 in Brisbane, Australia, will be organized by the HGSA on behalf of the IFHGS.

For Genetics in Medicine's new section on International Genetics, representatives of all Corresponding Member Societies were invited to contribute a short article or editorial about activities and developments in human and medical genetics in their country. The first two responses, from the Portuguese Society of Human Genetics and the Hellenic Association of Medical Geneticists, are published on pages 220-221 of this issue, and others are in preparation. We hope that this will encourage the representatives of other Member Societies to follow suit.

While operating in environments that are politically and culturally different, we all can learn from each other's experiences. Issues that are shared include delivery of genetic services and reimbursement; recognition of medical genetics as a specialty; education of medical students, physicians, and the public; funding for genetics research; access to the electronic scientific literature; participation of human subjects in research; and genetic privacy. There will also be issues that are specific to individual countries or regions. Population frequencies of disease genes vary, and otherwise rare disorders may be common in one region due to specific founder mutations, such as Machado-Joseph disease in Portugal.

We must meet the challenges together, by sharing information, technology, and resources. New avenues for communication and collaboration need to be established. It would be desirable to develop a consensus on policy matters of international concern. As commercial medical research organizations are crossing borders to recruit human subjects for large-scale genotyping studies, and human cloning enterprises are being set up abroad, a need for international ethics standards arises. Consensus policy statements and opinions will need to be prepared and transmitted to appropriate governmental and other regulatory agencies to ensure fair and ethical practices in the study of human genome diversity in health and disease. An agenda for International Genetics is taking shape.

Given the rapid dissemination of new information on the Internet (witness the speed with which a computer virus spreads!), publication of these International Genetics reports in a print journal is augmented by posting them, with free access, at http://www.ifhgs.org, thanks to the generosity of the editor and publisher of Genetics in Medicine.

Uta Francke, MD

Professor of Genetics and Pediatrics Stanford University, Stanford, California President (2000-2002)

International Federation of Human Genetics Societies 